\title{
Rotating Space Fabric of Universe Responsible for Expansion of Universe
}

\author{
Bhushan Bhoja Poojary \\ BSE Physics, NIMS University, Jaipur, India \\ Email address: \\ bhushanpoojary@gmail.com
}

\section{To cite this article:}

Bhushan Bhoja Poojary. Rotating Space Fabric of Universe Responsible for Expansion of Universe. American Journal of Astronomy and Astrophysics. Vol. 4, No. 4, 2016, pp. 38-41. doi: 10.11648/j.ajaa.20160404.11

Received: June 19, 2016; Accepted: July 4, 2016; Published: July 18, 2016

\begin{abstract}
Unknown force dark energy is linked to expansion of universe; this force can be understood by considering fabric of spacetime of entire universe in rotation. When a body moves in curved path in flat spacetime it experiences centrifugal force which pushes body outwards, similarly if a body in rest and spacetime fabric is in rotation around this body, body will accelerate in outward direction.
\end{abstract}

Keywords: Dark Energy, Black Hole, Vortex, Dark Flow, Holographic Principle

\section{Introduction}

Unknown force dark energy is linked to expansion of universe; this force can be understood by considering fabric of spacetime of entire universe in rotation. When a body moves in curved path in flat spacetime it experiences centrifugal force which pushes body outwards, similarly if a body in rest and if spacetime fabric is in rotation around this body, body will accelerate in outward direction. Rotating spacetime fabric with respect to cosmos explains the expansion of cosmos in all direction. Here we show direct evidences of spacetime fabric of universe is in rotational motion by using recent findings and research's, recently a research articles proved jets of black holes in center of galaxies are mysteriously aligned (black holes rotation aligns with rotation of spacetime fabric of universe which explains this phenomena) and dark flow phenomenon which is unknown force which is pulling galaxy clusters towards a particular point in space (galaxy cluster are moving towards the edge of the universe due to rotating spacetime fabric).

\section{Rotating Fabric of Space of Universe}

The holographic principle states that the description of a volume of space can be thought of as encoded on a lower-dimensional boundary to the region-preferably a light-like boundary like a gravitational horizon [1]. The theory in short says entire universe is encoded in 2 dimension on the cosmological horizon, the event horizon from which information may still be gathered and not lost due to the natural limitations of spacetime supporting a black hole, an observer and a given setting of these specific elements, such that the three dimensions we observe are an effective description only at macroscopic scales and at low energies

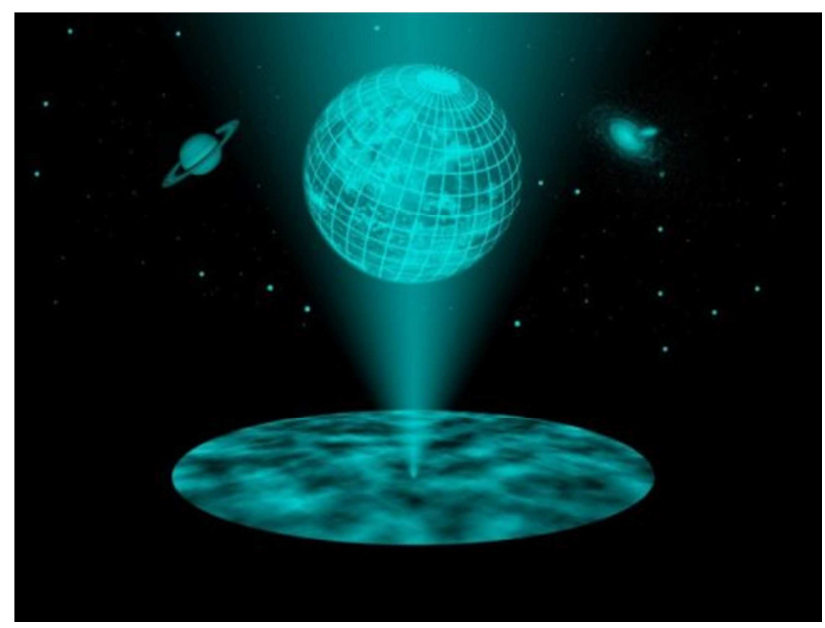

Figure 1. Holographic principle credit TU Wien.

As entire information is encoded in cosmological horizon, 
fabric of space which surrounds every element in the universe too is encoded in this cosmological horizon. All black holes rotate in very high speed (this is because black holes are formed when there is gravitational collapse of massive stars), as black hole rotates, cosmological horizon too should rotate with it, as fabric of space is projection of the information encoded in cosmological horizon, fabric of entire universe should rotate.

\section{Hubble Law Analogy to Circular Motion}

Hubble's law is considered a fundamental relation between recessional velocity and distance.

Hubble law is given as [2]

$$
v=H_{o} D
$$

Where $v$ is recessional velocity, which is with how much velocity the object is receding from the observer.

$H_{o}$ is Hubble's constant, which is ratio of change in acceleration to acceleration of galaxy with respect to observer.

$D$ is proper distance between observer and galaxy.

Hubble constant is given as

$$
H_{o}=\frac{\dot{a}}{a}
$$

Where $a$ is acceleration of galaxy and $\dot{a}$ is change in acceleration.

Let us consider a flat disk rotating with constant angular velocity, suppose a marble is placed in this rotating disk, due to centrifugal force the marble will star moving towards the edge of the disc.

Acceleration ' $a$ ' of the marble of mass ' $m$ ' would be if the marble is at distance ' $r$ ' away from the center.

$$
a=m r \omega^{2}
$$

As marble moves away from the center acceleration will increase, so change in acceleration $\dot{a}$ can be given as

$$
\dot{a}=m \omega^{2} \frac{d r}{d t}
$$

$\frac{d r}{d t}$ can be considered as recessional velocity $v$, putting this value in Eq 4 we get

$$
\dot{a}=m \omega^{2} v
$$

If we divide $\mathrm{Eq} 5$ and $\mathrm{Eq} 3$ we get

$$
\frac{\dot{a}}{a}=\frac{v}{r}
$$

Comparing Eq6 with Eq1, we can see they are same, and can conclude that Hubble law is related to some kind of circulation motion of cosmological horizon, which projects this rotating $2 \mathrm{D}$ information in our $3 \mathrm{D}$ physical universe.

\section{Evidences That Our Universe Is Inside a Rotating Fabric of Spacetime}

\subsection{Alignments of Radio Galaxies in Deep Radio Imaging of ELAIS N1)}

Einstein predicted that the spinning earth should create a vortex of spacetime near it (as massive objects rotate it should drag the space fabric around it), which was confirmed by NASA by using results of Gravity Probe B (GP-B) [3]. In this experiment, a gyroscope experiences a drag due to this vortex. Scientist measured the shift in gyroscope and figured out that it matched Einstein general theory of relativity prediction.

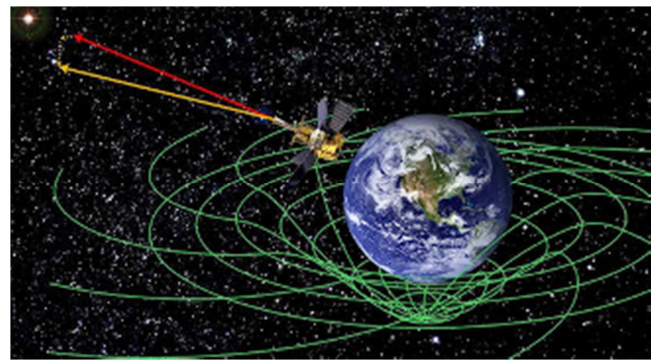

Figure 2. An artist's concept of GP-B measuring the curved spacetime around Earth.

Considering our universe in kind of vortex (rotating spacetime fabric), objects would experience a similar drag and move away from each other. As this is intrinsic property of spacetime one cannot see the source of the energy, hence it seems like an illusion of some unknown force which is responsible for expansion of universe as a drag due to this vortex. There's a region in the distant Universe where a few supermassive black holes have mysteriously aligned [4]. These supermassive black holes act similar to gyroscope used in Gravity Probe B (GP-B), rotation of these black holes get shifted due to vortex of spacetime of universe and as these black holes arelwere relatively closer to each other, their axis of spins aligns in same direction.
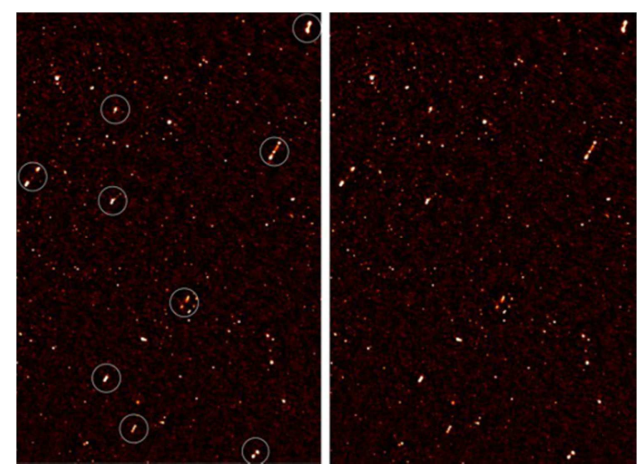

Figure 3. An image of the deep radio map covering the ELAIS-N1 region, with aligned galaxy jets. The image on the left has white circles around the aligned galaxies; the image on the right is without the circles. Image credits: Prof. Russ Taylor.

\subsection{Dark Flow}

The Dark flow is phenomena where group of galaxy 
clusters moving towards a point of space known as Great attractor [5]. Considering a spinning sphere of water in no gravity with suspended heavy particles within it, all particles will initially move outwards and merge and collect at the rim where the speed is maximum due to centrifugal force. Similarly, considering vortex of universe, all galaxy cluster should start moving towards the edge of the universe, where speed of the vortex is maximum.

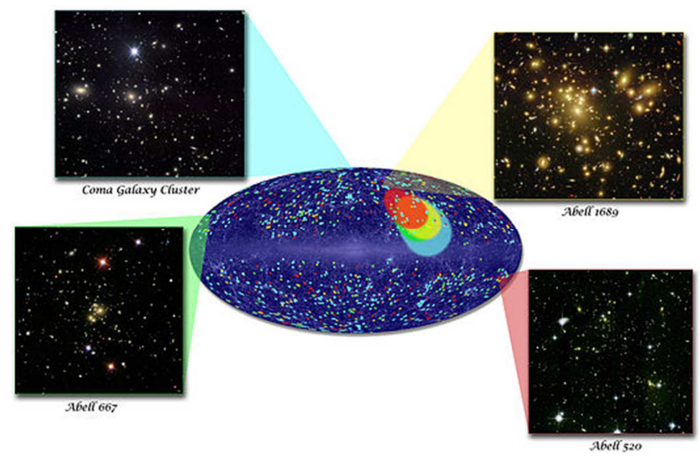

Figure 4. The dark flow. The colored dots are clusters within one of four distance ranges, with redder colors indicating greater distance. Colored ellipses show the direction of bulk motion for the clusters of the corresponding color. Images of representative galaxy clusters in each distance slice are also shown. Image credit: A. Kashlinsky (NASA).

\subsection{Ecliptic Alignment of CMB Anisotropy}

Motion of our solar system and plane of ecliptic (axis of evil [6]) are aligned with the features of microwave sky [7], this contradicts the Copernican principle. There is no conclusive proof provided for this alignment and it is believed it may be due to coincidence but the probability is extremely low. Considering whole universe fabric of space is rotating, our solar system tilted in direction of motion due to its relative motion with respect to rotating vortex of universe. Thus it is not mere coincidence for our solar system plane of axis matches line of evil. This also tells us line of evil is axis of rotation of our universe, and because universe was rotating, $\mathrm{CMB}$ is not homogeneous as predicted but there are anomalies in CMB [8] [9] which is big bang afterglow as measured by plank mission [10].

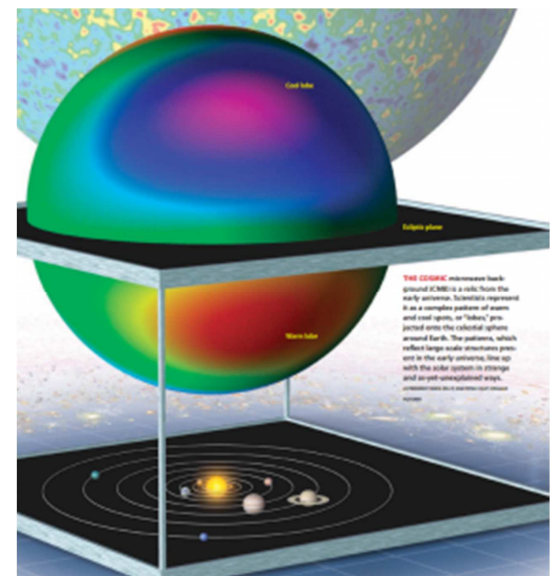

Figure 5. Alignment of Axis of Evil (Image Credit: Roen Kelly, Dragan Huterer).

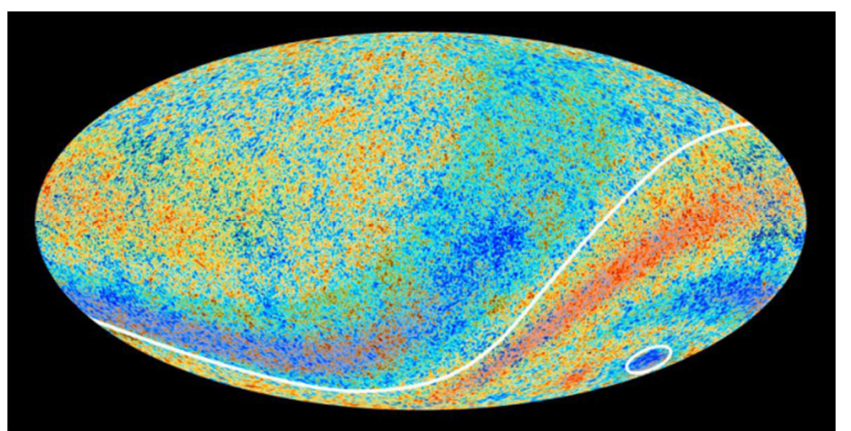

Figure 6. New observations with ESO's Very Large Telescope (VLT) in Chile have revealed alignments over the largest structures ever discovered in the Universe.

Which means universe was rotating since it was born.

Thus rotation of fabric of space of universe also explains alignment of our solar system axis with line of evil as well as anomalies found in CMD data.

\subsection{Spooky Alignment of Quasars Across Billions of Light-Years}

A team led by Damien Hutsemékers from the University of Liège in Belgium used the FORS instrument on the VLT to study 93 quasars that were known to form huge groupings spread over billions of light-years, seen at a time when the Universe was about one third of its current age [11].

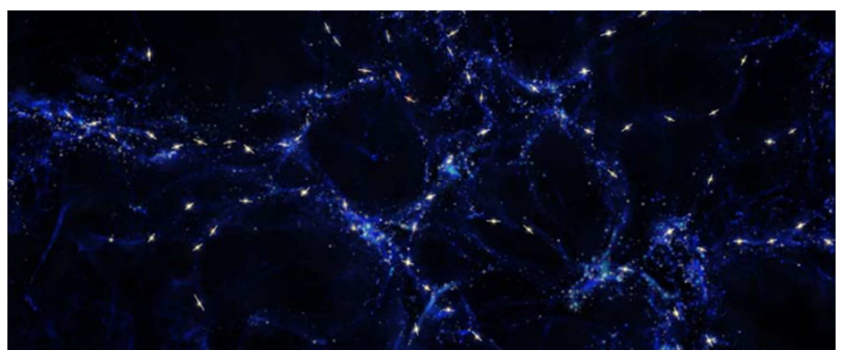

Figure 7. New observations with ESO's Very Large Telescope (VLT) in Chile have revealed alignments over the largest structures ever discovered in the Universe.

Probability of this alignment is only $1 \%$ and there is no proper explanation for such mysterious alignment. Space time of fabric of universe when viewed as rotating against such structure, this massive structure and vortex around it get influenced by each other. The flow of space time fabric varies close to this massive structure, as flow is consistent within close range of the massive structures, super massive black holes aligns as per the flow of fabric of space vortex as explained previously. As these black holes are at very far distance their magnetic properties should not have much impact or influence on each other, rotation of fabric of space combination of movement of massive structure creates such kind of alignment.

\subsection{Expansion of Universe Appears to Accelerate}

Universe is expanding and its expansion is speeding up [12], if you consider a sphere of water, rotating with suspended heavy particle in zero gravity, as the particle gets pushed 
towards the outward direction, centrifugal force acting on it will increase as acceleration is directly proportional to distance from center in case of circular motion. Which means as objects start moving away from the center, these objects will feel more centrifugal force and thus such objects will start accelerating faster. Thus rotation of fabric of space also explains linear increase in expansion of universe with respect to distance as acceleration is directly proportional to distance of object from center of rotation.

\section{Conclusion}

Dark energy is always being a mysteries force, source of this force is not a physical object in universe as most of the people expected and nor do physical universe (space itself expands) expand like balloon, main reason behind expansion is projection of rotating cosmological horizon (holographic principle), this projection rotates the fabric of entire universe in circular motion. As fabric rotates, objects within it feel the centrifugal force due to rotation. Due to this centrifugal force objects starts appearing as expanding.

\section{References}

[1] Susskind, Leonard (1995). "The World as a Hologram". Journal of Mathematical Physics 36 (11): 6377-6396. arXiv:hep-th/9409089. Bibcode:1995JMP....36.6377S. doi: $10.1063 / 1.531249$.

[2] Riess, A.; et al. (September 1998). "Observational Evidence from Supernovae for an Accelerating Universe and a Cosmological Constant". The Astronomical Journal 116 (3): 1009-1038.arXiv:astro-ph/9805201.

Bibcode:1998AJ...116.1009R. doi: 10.1086/300499.
[3] Gravity Probe B Testing Einstein's Universe https://www.nasa.gov/pdf/162789main_gpb_fs.pdf

[4] Alignments of radio galaxies in deep radio imaging of ELAIS $\mathrm{N} 1$ http://mnrasl.oxfordjournals.org/content/459/1/L36

[5] A. Kashlinsky, F. Atrio-Barandela, D. Kocevski, and H. Ebeling (2008). "A measurement of large-scale peculiar velocities of clusters of galaxies: results and cosmological implications" (PDF). Astrophys. J. 686: 49-52. arXiv:0809.3734. Bibcode:2008ApJ...686L..49K. doi: 10.1086/592947. Retrieved 2010-07-15.

[6] Zeeya Marili (2007-04-13). "'Axis of evil' a cause for cosmic concern". New Scientist. Archived from the original on May 21, 2007. Retrieved 2007-05-22.

[7] C. J. Copi, D. Huterer, D. J. Schwarz, G. D. Starkman (2006). "On the large-angle anomalies of the microwave sky". Monthly Notices of the Royal Astronomical Society 367: 79-102.

[8] Mariano, Antonio; Perivolaropoulos, Leandros (2013). "CMB maximum temperature asymmetry axis: Alignment with other cosmic asymmetries". Physical Review D 87 (4). doi: 10.1103/PhysRevD.87.043511. ISSN 1550-7998.

[9] Rossmanith, G.; Räth, C.; Banday, A. J.; Morfill, G. (2009). "Non-Gaussian Signatures in the five-year WMAP data as identified with isotropic scaling indices". Monthly Notices of the Royal Astronomical Society 399 (4): 1921-1933. arXiv:0905.2854.Bibcode:2009MNRAS.399.1921R. doi: 10.1111/j.1365-2966.2009.15421.x.

[10] http://esamultimedia.esa.int/docs/planck/Planck-Factsheet.pdf Retrieved 2016-05-22

[11] https://www.eso.org/public/news/eso1438/ 2016-05-22

Retrieved

[12] http://www.space.com/17884-universe-expansion-speed-hubbl e-constant.html Retrieved 2016-05-22 